\title{
Field galaxies at intermediate redshift $(0.2<z<0.8)$ in the direction of the galaxy cluster LCDCS-S001
}

\author{
A. C. Krabbe, ${ }^{\star}$ S. B. Rembold and M. G. Pastoriza \\ Instituto de Física, Universidade Federal do Rio Grande do Sul, Av. Bento Gonalves, 9500, Cep 91359-050, Porto Alegre, RS, Brazil
}

Accepted 2007 March 23. Received 2007 March 21; in original form 2006 December 29

\begin{abstract}
We present a spectroscopic and photometric analysis for eight field galaxies in the direction of the galaxy cluster LCDCS-S001. The spectra were obtained with the GMOS instrument at the Gemini South Observatory. The objects were selected in an $i^{\prime}$-band image and the multiobject spectroscopic observations were centred at $7500 \AA$. For the galaxies ID 440 and ID 461, we have determined redshifts of $z=0.7464$ and 0.7465 , respectively. For the other six galaxies we have confirmed the previous measurements of redshift. The redshifts of the field galaxies are in the range of $0.2201<z<0.7784$. We determined the blue and visual luminosities and they are brighter than $M_{B}=-18.64$. The galaxies ID 180, ID 266 and ID 461 follow the Faber-Jackson relation of the Coma and Virgo early-type galaxies, and therefore do not present a brightening of the $B$ luminosity as observed in galaxies at higher redshifts. The stellar velocity dispersion was measured for five galaxies (ID 146, ID 180, ID 266, ID 428 and ID 440) and estimated to be in the range of $200<\sigma<346 \mathrm{~km} \mathrm{~s}^{-1}$. Lick indices were measured and used to determine the stellar population properties of the galaxies ID 120 and ID 146, by means of spectral synthesis. The first galaxy, ID 120, presents in its spectrum absorption and emission lines, and we have found that the main contribution in the flux at $\lambda 5870 \AA$ is of a $0.1-$ Gyr stellar population of solar metallicity. For ID 146, the dominant flux contribution at $\lambda 4200 \AA \AA$ is provided by a stellar population of $10 \mathrm{Gyr}$ of subsolar metallicity. From stellar population synthesis, we estimated reddening values of $E(B-V)=0.90$ and 0.82 for ID 120 and ID 146, respectively. According to classical diagnostic diagrams the emission lines present in the spectrum of ID 120 indicate that it is a starburst galaxy.
\end{abstract}

Key words: galaxies: clusters: general - galaxies: stellar content.

\section{INTRODUCTION}

Galaxy clusters are one of the fundamental sites to study the formation and evolution of galaxies. Many observational studies in cluster galaxies have revealed that galaxy properties such as colours, morphology and stellar populations vary at different redshifts. Butcher \& Oemler (1984) have found in intermediate-redshift clusters that the fraction of blue galaxies (Butcher \& Oemler 1984) and galaxies with star formation (Poggianti et al. 1999) is higher than that in clusters of the local Universe. Luminosity evolution was also observed for galaxies at intermediate redshift with respect to the local galaxies. There was a brightening in blue magnitude in the Faber-Jackson relation (Ziegler \& Bender 1997; Fritz et al. 2005).

Differences between the stellar population of cluster and field galaxies at intermediate and higher redshifts have been revealed. Serote Roos et al. (2005) have found that the field galaxies seem to host less-evolved stellar populations than cluster members, and

${ }^{\star}$ E-mail: angela.krabbe@ufrgs.br van Dokkum et al. (2001) found that the field early-type galaxies are about 20 per cent younger than cluster early-type galaxies. Moreover, significant offsets between field and cluster galaxies were derived at $z=0.7$ by Treu et al. (2002). However, few observational works on clusters and field galaxies at intermediate redshifts have been published to confirm these results.

In a previous work, Rembold \& Pastoriza (2007) (hereafter Paper I) presented a study on the kinematic parameters and stellar population properties of galaxies of cluster LCDCS-S001 $(z=$ $0.7)$. In the field of view of this galaxy cluster several galaxies were observed at intermediate redshifts which are not cluster members. In particular, ID 120 at $z=0.2201$ shows in its spectrum very bright emission lines apparently typical of starburst galaxies. We have studied these galaxies and report in this paper the more important spectroscopic and photometric results, such as redshifts, stellar population, blue luminosity and for ID 120 we discuss the properties of the ionized gas. The paper is structured as follows. In Section 2, we describe the observations and data reduction. In Section 3, we present the determination of redshifts and velocity dispersions. In Section 4, we describe the determination of absolute magnitudes and 
Table 1. Coordinates, redshifts, absolute magnitudes and spectral lines of the observed galaxies.

\begin{tabular}{|c|c|c|c|c|c|c|c|c|c|}
\hline ID & 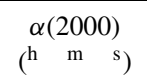 & $\begin{array}{l}\delta(2000) \\
\left(\begin{array}{lll}\mathrm{h} & \mathrm{m} & \mathrm{s}\end{array}\right)\end{array}$ & $z$ & $\begin{array}{c}\sigma \\
\left(\mathrm{km} \mathrm{s}^{-1}\right)\end{array}$ & $i_{A B}^{\prime}$ & $M_{A B, i^{\prime}}$ & $M_{B}$ & $M_{V}$ & Spectral lines \\
\hline 120 & 100625.20 & -125856.6 & 0.2201 & $\ldots$ & 21.42 & -18.75 & $\ldots$ & -18.20 & $\begin{array}{l}\mathrm{CH} \lambda 4300, \mathrm{H} \beta \lambda 4861,[\mathrm{O} \text { III] } \lambda 4959,[\mathrm{O} \text { III] } \lambda 5007, \\
\mathrm{Mg}_{\mathrm{I}} \lambda 5167, \mathrm{NaD} \lambda 5890,\left[\mathrm{~N}_{\mathrm{II}}\right] \lambda 6548, \mathrm{H} \alpha \lambda 6563, \\
{\left[\mathrm{~N}_{\mathrm{II}}\right] \lambda 6584,\left[\mathrm{~S}_{\mathrm{II}}\right] \lambda 6717,\left[\mathrm{~S}_{\mathrm{II}}\right] \lambda 6731}\end{array}$ \\
\hline 146 & 100621.10 & -125907.7 & 0.3910 & $217 \pm 19$ & 21.50 & -20.11 & -18.64 & .. & $\begin{array}{l}\mathrm{Fe} \text { I } \lambda 3631, \mathrm{Fe} \text { I } \lambda 3720, \mathrm{Fe} \text { I } \lambda 3827, \mathrm{H} 9 \lambda 3835, \\
\mathrm{Ca} \text { II } \lambda 3934, \mathrm{Ca} \text { II } \lambda 3968, \mathrm{H} \delta \lambda 4101, \mathrm{Ca} \text { I } \lambda 4226, \\
\mathrm{CH} \lambda 4300, \mathrm{Fe} \text { I } \lambda 4383, \mathrm{H} \beta \lambda 4861,\left[\mathrm{~N}_{\mathrm{I}}\right] \lambda 5200\end{array}$ \\
\hline 180 & 100614.90 & -125907.9 & 0.6508 & $274 \pm 58$ & 21.75 & -21.20 & -20.66 & .. & Ca II $\lambda 3934$, Ca II $\lambda 3968, \mathrm{CH} \lambda 4300, \mathrm{H} \gamma \lambda 4340$ \\
\hline 237 & 100619.22 & -125817.0 & 0.2607 & $\ldots$ & 20.82 & -19.46 & $\ldots$ & -18.93 & $\mathrm{H} \beta \lambda 4861,[\mathrm{O} \mathrm{III]}] \lambda 5007$ \\
\hline 266 & 100615.80 & -125828.8 & 0.7784 & $200 \pm 23$ & 21.89 & -21.54 & -21.29 & $\ldots$ & $\begin{array}{l}\text { H10 } \lambda 3798, \text { H9 } \lambda 3835, \text { H8 } \lambda 3889, \text { Ca II } \lambda 3934 \text {, } \\
\text { Ca II } \lambda 3968\end{array}$ \\
\hline 428 & 100617.14 & -125641.6 & 0.7784 & $278 \pm 89$ & 21.82 & -22.08 & -22.45 & $\ldots$ & Ca II $\lambda 3934$, Ca II $\lambda 3968$ \\
\hline 440 & 100614.71 & -125644.8 & 0.7464 & $346 \pm 12$ & 20.98 & -22.34 & -22.01 & $\ldots$ & Са II $\lambda 3934$, Ca II $\lambda 3968$ \\
\hline 461 & 100611.24 & -125710.8 & 0.7465 & $\ldots$ & 20.47 & -22.85 & -22.52 & $\ldots$ & Са II $\lambda 3934$, Ca II $\lambda 3968$ \\
\hline
\end{tabular}

Conventions. $\alpha, \delta$ : equatorial coordinates.

the relation between luminosity and velocity dispersion. Section 5 deals with the stellar population synthesis, describing the measurements of Lick indices, the method used and the results obtained. In Section 6 , we determine the properties of the nebular gas and the nature of the ionization source. The conclusions are given in Section 7. Throughout this paper we adopt a cosmological model with $H_{0}=71 \mathrm{~km} \mathrm{~s}^{-1}, \Omega_{\mathrm{M}}=0.27$ and $\Omega_{\mathrm{V}}=0.73$.

\section{OBSERVATIONS AND DATA REDUCTIONS}

This paper is based on spectroscopic data obtained in 2004 March with the Gemini Multi-Object Spectrograph (GMOS) of the Gemini South Observatory. The GMOS mask was built using as reference an $i^{\prime}$-filter image obtained with GMOS, which covered an area of $330 \times$ $330 \operatorname{arcsec}^{2}$, with a total integration time of $300 \mathrm{~s}$. The detector was binned at $2 \times 2$ pixels, giving a spatial scale of $0.146 \operatorname{arcsec~pixel}^{-1}$. The objects included in this study are listed in Table 1. We used a grid of 400 grooves $\mathrm{mm}^{-1}$. The spectra were obtained in the 5560 $9720 \AA$ range with a dispersion of $0.67 \AA$ pixel $^{-1}$ and a spectral resolution of $R \simeq 1918$. Two masks were needed to include all observed objects. The exposure time for each mask was limited to $1200 \mathrm{~s}$ to minimize the effects of cosmic rays and to obtain a signal-to-noise ratio higher than 3 .

The data reduction followed the standard procedures of bias correction, flat-fielding, cosmic-ray cleaning, sky subtraction, wavelength and flux calibrations, and one-dimensional spectrum extraction, and was made using mainly the IRAF software. The procedures we followed in the data reduction are described in detail in Paper I. Fig. 1 shows the full area imaged by GMOS and the analysed objects, which are numbered and marked with the circles. Fig. 2 shows the isophotal maps in the $i^{\prime}$ band for each galaxy of our sample. In Fig. 3, we present one-dimensional spectra where the most prominent lines are identified and listed in Table 1.

\section{DETERMINATION OF REDSHIFTS AND VELOCITY DISPERSIONS}

The spectroscopic redshifts for ID 120, ID 146, ID 180, ID 237 , ID 266 and ID 428 were taken from Paper I. In this work we determined the redshift only for the galaxies ID 440 and ID 461. The redshift of these galaxies was calculated by the average recession velocity given by the individual lines and estimated to be around $z=$

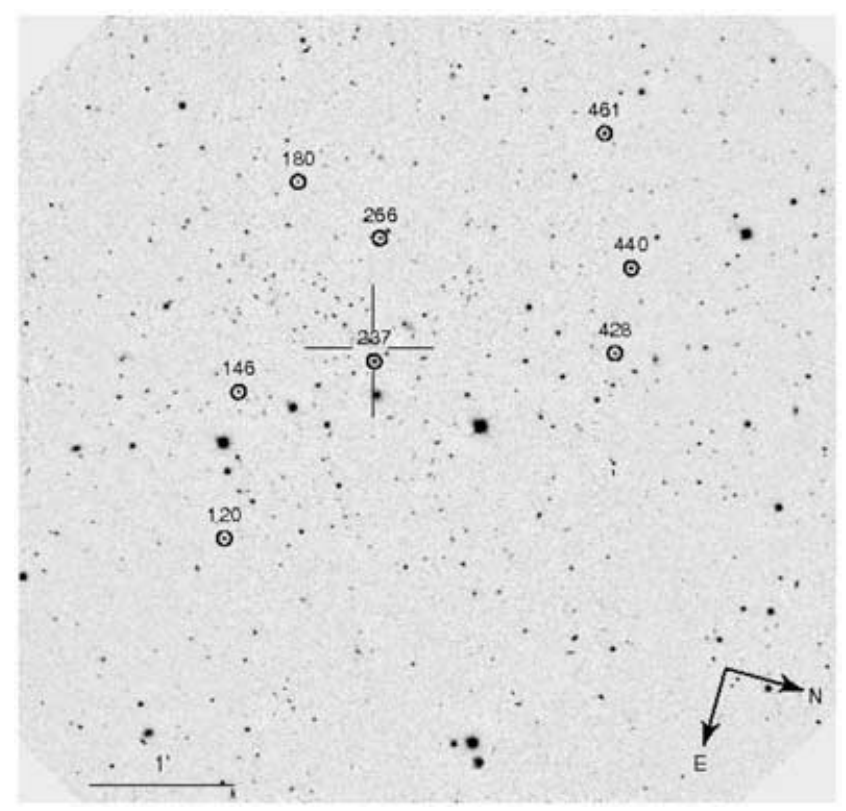

Figure 1. $i^{\prime}$-band image of the galaxy cluster LCDCS-S001 with the field galaxies marked with the circles and the visual centre of the cluster marked with a cross.

0.7464 and 0.7465 for ID 440 and ID 461, respectively. The galaxy cluster LCDCS-S001 has a median cluster redshift of 0.709 and an estimated velocity dispersion of $\sigma=727 \mathrm{~km} \mathrm{~s}^{-1}$ (see Paper I); therefore, the last galaxies are not cluster members. Note that the galaxies of our sample can be separated into two sets: one (ID 120, ID 146, ID 237) located to the east of the cluster centre, in the redshift range $0.2201<z<0.3910$, and the other set of galaxies (ID 266, ID 428, ID 440, ID 461) is to the west of the centre and the redshift is in the range $0.7464<z<0.7784$. The galaxy ID 180 to the west of the cluster centre is at redshift $z=0.6508$. The errors associated with the redshift for each galaxy, estimated from the standard deviation of the individual redshifts, are practically negligible, below 1 per cent.

The velocity dispersions, $\sigma$, of the observed galaxies were determined from the measurements of corrected full width at halfmaximum (FWHM), assuming Gaussian profiles for the absorption 

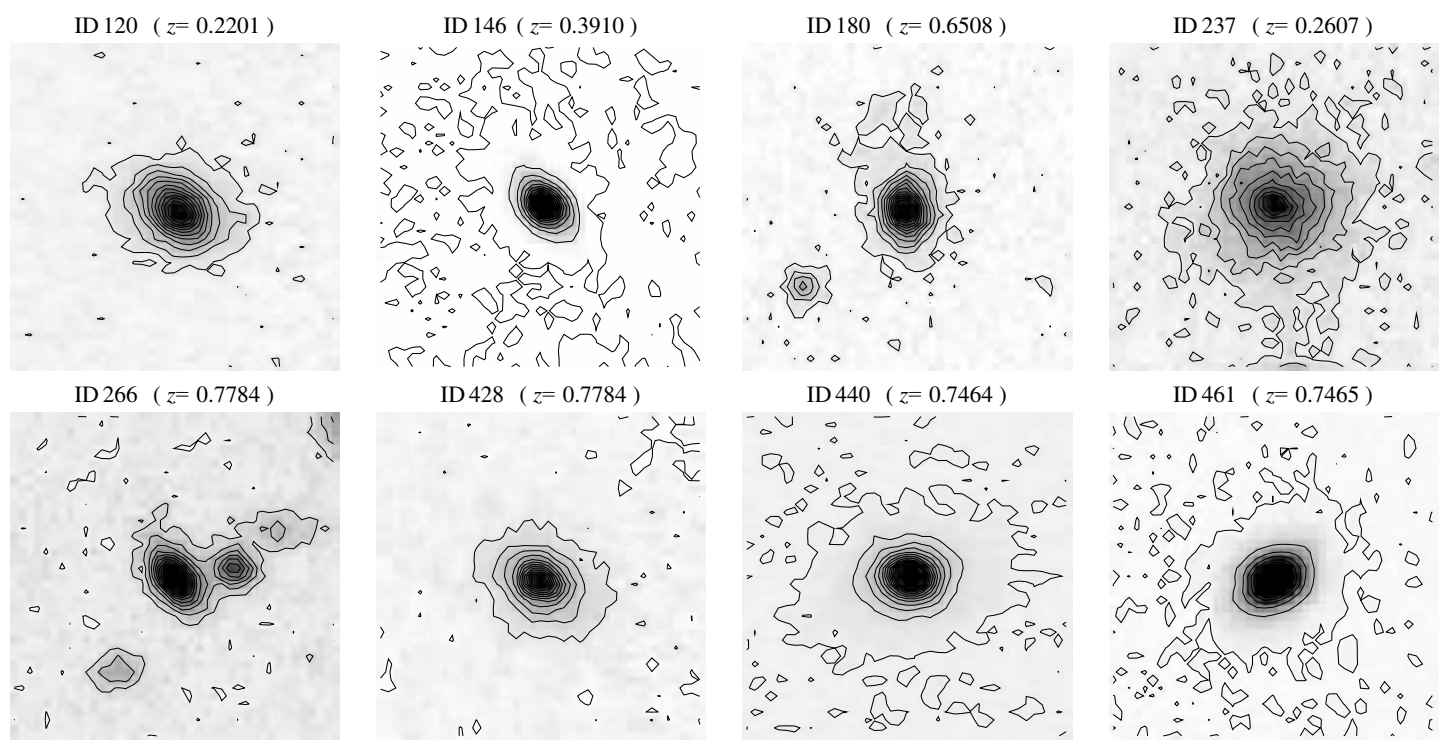

Figure 2. $i^{\prime}$-band images and contours of the observed galaxies. All frames are $5.99 \times 5.99 \operatorname{arcsec}^{2}$.

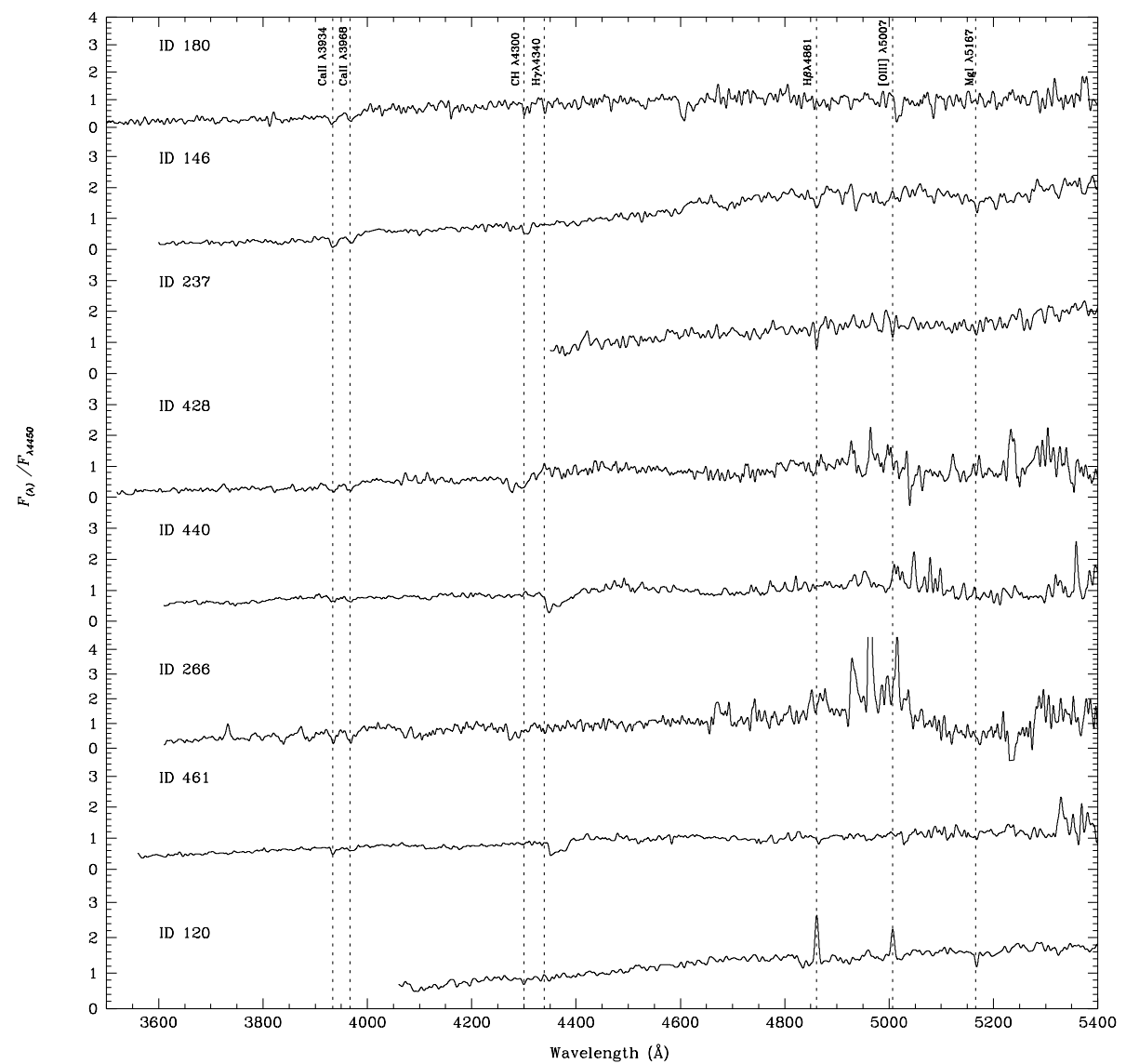

Figure 3. Spectra of the field galaxies in the range of 3500 to $5400 \AA$. The most prominent absorption and emission lines are marked.

lines. The effects of the instrumental dispersion and resolution on the observed FWHM were estimated from the measurements of the absorption lines Ca II $\lambda 8542$ and Ca II $\lambda 8662$ observed in the spectrum of a $\mathrm{G}$ star identified in the field. We have found that the velocity dispersions for most of our galaxies (ID 146, ID 180,
ID 266, ID 428, ID 440) are in the range $200<\sigma<346 \mathrm{~km} \mathrm{~s}^{-1}$. The galaxies ID 120, ID 237 and ID 461 have velocity dispersions of the order of the instrumental resolution and therefore are not estimated. The values of redshift and velocity dispersions $\sigma$ are listed in Table 1. 


\section{DETERMINATION OF ABSOLUTE MAGNITUDES}

In this paper, we have estimated the absolute magnitudes $M_{B}$ and $M_{V}$ for our sample of galaxies using the values of $i_{A B}^{\prime}$ photometric magnitude taken from Paper I and the $B$ and $V$ magnitudes derived from the observed spectra. In order to calculate these magnitudes, we have calibrated the observed spectra using the $i_{A B}^{\prime}$ photometric magnitude. Note that the GMOS filters are defined in the $A B$ system as

$i_{A B}^{\prime}=-2.5 \log _{10} f_{v}\left(\mathrm{erg} \mathrm{s}^{-1} \mathrm{~cm}^{-2} \mathrm{~Hz}^{-1}\right)-48.60$.

We have transformed the spectra to the rest-frame system and applied for the fluxes the $(1+z)^{2}$ correction for cosmological dimming. We have then calculated the $B$ and $V$ magnitudes from the spectra by integrating the fluxes through a set of filter response curves using the SBANDS routine of the ONEDSPEC package of the NOAO/IRAF. Finally, we added this magnitude to the distance modulus, calculated as a function of the galaxy redshift $z$ adopting the cosmological model assumed in this paper. The values of $M_{B}, M_{V}$ and $M i_{A B}^{\prime}$ (taken from Paper I) are listed in Table 1 . We verify that our field galaxies display a correlation between redshift and absolute magnitude in $i_{A B}^{\prime}$ band as well as in the $V$ band. This means that high-luminosity galaxies are detected at higher redshift, while low-luminosity galaxies are detected mainly at lower redshifts. This behaviour is a selection effect for samples that are chosen based on magnitudes. The total error associated with the absolute magnitudes was estimated to be in the range of 0.12 to $0.37 \mathrm{mag}$. This error was estimated considering (i) the uncertainties due to the continuum signal-to-noise ratio in each spectrum and (ii) the uncertainties of the $i^{\prime}$ magnitude, which are mainly due to errors on the count fluctuations, the assumption of the zero-point and the lack of a colour term correction.

The field galaxies in our sample are very luminous with blue luminosity in the range of $-18.64<M_{B}<-22.52$. Their luminosities are comparable with the luminosities of galaxies at intermediate redshifts (Ziegler \& Bender 1997; Mouhcine et al. 2006; Nakamura et al. 2006).

\section{FABER-JACKSON RELATION}

It is well known that elliptical and lenticular galaxies follow the scaling relation between galaxy luminosity and velocity dispersion (Faber \& Jackson 1976), which is widely used to obtain important information about elliptical galaxies. This is called the Faber-Jackson relation and is valid both for local- and for intermediate-redshift galaxies. We searched in our sample if there are early-type galaxies that follow this relation and present a brightening of blue magnitude with respect to local clusters of galaxies as inferred by Ziegler \& Bender (1997) .

In Fig. 4, the $M_{B}$ versus $\sigma$ relation for the galaxies ID 146, ID 180, ID 266, ID 428 and ID 440 is plotted and also compared with data of a sample of Coma and Virgo elliptical galaxies (Dressler et al. 1987) and elliptical galaxies of clusters at $z=0.37$ (Ziegler $\&$ Bender 1997). As can be seen in this figure, the galaxies ID 180, ID 266, ID 428 and ID 440 follow the Faber-Jackson relation indicating that they are early-type galaxies. The isophote maps of the first three galaxies are typical of ellipticals. In the case of ID 440 the isophotal map shows an extended structure probably due to the presence of a disc; therefore, this galaxy may be lenticular. Deep imaging with higher spatial resolution is necessary to confirm the morphology of these galaxies.

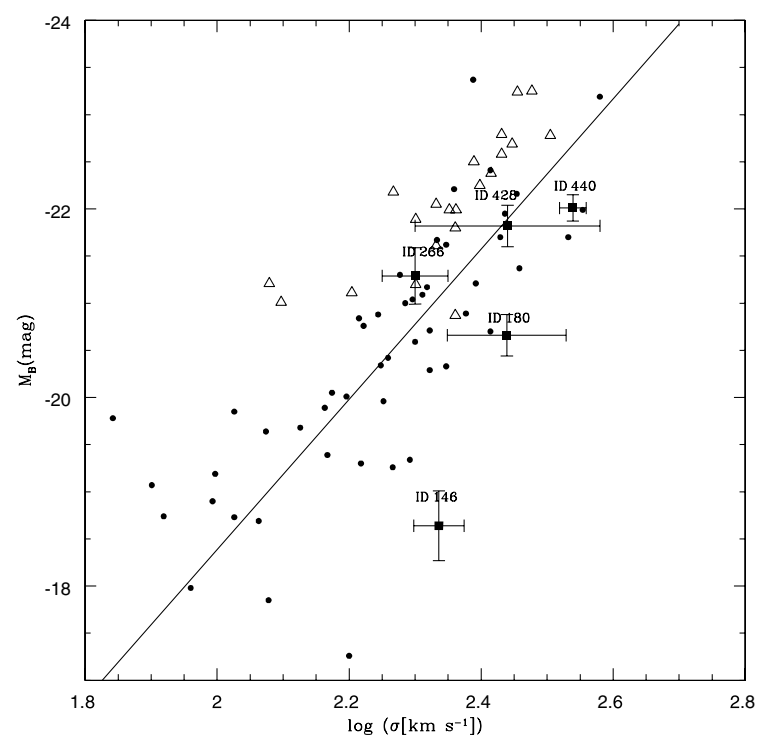

Figure 4. Faber-Jackson relation in absolute $B$ magnitude for five galaxies in the field of the galaxy cluster LCDCS-S001(square with error bars). The circles represent the Coma and Virgo sample of Dressler et al. (1987) and the triangles represent the elliptical galaxy sample at $z=0.37$ of Ziegler \& Bender (1997). The solid line is the best fit for the Coma sample, as derived by Ziegler \& Bender (1997).

The galaxy ID 146 follows the Faber-Jackson relation marginally, that is, a difference of about 2 mag is present between this galaxy and the derived fit by Ziegler \& Bender (1997) for Coma galaxies. Also, it can be seen in Fig. 2 that the more external isophote shows an extended structure. Therefore, this galaxy probably has a morphology intermediate between lenticular and early spiral. The remaining galaxies ID 120, ID 237 and ID 461 have velocity dispersions of the order of the instrumental resolution and due to their isophotal map morphology they might be spiral galaxies.

The most important result obtained in this section is that the earlytype field galaxies (ID 146, ID 180, ID 266, ID 428 and ID 440) have velocity dispersions and luminosities comparable to those of members of Coma and Virgo clusters and do not present a brightening of blue magnitude as observed by Ziegler \& Bender (1997) for elliptical galaxies at $z=0.37$.

\section{STELLAR POPULATION SYNTHESIS}

We have performed the stellar population synthesis method developed by Bica (1998), in order to describe the age distribution of the stellar population of these galaxies and get some insights about their metallicity. This synthesis method uses the equivalent widths $W_{\lambda}$ of spectral absorption features and measured continuum fluxes $F_{\lambda}$ at a given wavelength and compares them to the same quantities measured from a base of simple stellar population elements, which in turn have known ages and metallicities. The algorithm used is based on an upgraded version of Schmitt, Bica \& Pastoriza (1996), which also corrects the effect of the internal extinction that affects the stellar population.

The employed $W_{\lambda}$ were those defined by the Lick system (Worthey \& Ottaviani 1997; Trager et al. 1998). Previous to the measurements of the $W_{\lambda}$ and $F_{\lambda}$ the spectra have been normalized at $5870 \AA$ for ID 120 and at $4200 \AA$ for ID 146 and ID 180. The $W_{\lambda}$ values for each galaxy are listed in Table 2 . The base elements that we have used were taken from the Bruzual \& Charlot (2003) 
Table 2. Lick indices.

\begin{tabular}{lccc}
\hline Equivalent width $(\AA)$ & ID 120 & ID 146 & ID 180 \\
\hline $\mathrm{H} \delta$ & $\ldots$ & $2.56 \pm 0.13$ & $\ldots$ \\
$\mathrm{Ca}$ I $\lambda 4227$ & $\ldots$ & $1.57 \pm 0.14$ & $\ldots$ \\
$\mathrm{G} \lambda 4300$ & $1.324 \pm 0.13$ & $5.64 \pm 1.13$ & $6.67 \pm 0.67$ \\
$\mathrm{H} \gamma \lambda 4340$ & $\ldots$ & $\ldots$ & $2.91 \pm 0.87$ \\
$\mathrm{H} \beta \lambda 4861$ & $\ldots$ & $4.20 \pm 0.50$ & $\ldots$ \\
$\mathrm{Mg} \mathrm{I} \lambda 5167$ & $1.70 \pm 0.43$ & $\ldots$ & $\ldots$ \\
$\mathrm{NaD} \lambda 5890$ & $2.76 \pm 0.44$ & $10.14 \pm 0.61$ & $\ldots$ \\
\hline
\end{tabular}

Table 3. Stellar population synthesis results.

\begin{tabular}{lccc}
\hline Age (Gyr) & $\begin{array}{c}\text { ID 120 } \\
\lambda 5870 \text { flux fraction } \\
\text { (per cent) }\end{array}$ & $\begin{array}{c}\text { Age (Gyr) } \\
Z=0.004\end{array}$ & $\begin{array}{c}\text { ID 146 } \\
\lambda 4200 \text { flux fraction } \\
\text { (per cent) }\end{array}$ \\
\hline 0.1 & $46.64 \pm 2.91$ & 0.1 & $5.21 \pm 4.00$ \\
1 & $8.51 \pm 5.63$ & 1 & $18.89 \pm 4.88$ \\
5 & $13.56 \pm 9.10$ & 5 & $16.74 \pm 3.36$ \\
10 & $32.29 \pm 6.27$ & 9 & $59.16 \pm 2.91$ \\
$E(B-V)$ & 0.82 & $E(B-V)$ & 0.90 \\
\hline
\end{tabular}

evolutionary stellar population models, which are based on a highresolution library of observed stellar spectra. This library allows us to derive a detailed spectral evolution of simple stellar populations across the wavelength range of 3200 to $9500 \AA$ with a wide range of metallicities. We have used the Padova 1994 tracks as recommended by Bruzual \& Charlot (2003).

In this work, we derived the stellar population for ID $120(z=$ $0.22)$ and ID $146(z=0.39)$, the only galaxies in our sample with enough measured Lick indices that allow us to perform a reliable stellar population synthesis. Following the cosmological model adopted, the age of the Universe at redshifts $z=0.22$ and 0.39 is around 10 and $9 \mathrm{Gyr}$, respectively. 10 and $9 \mathrm{Gyr}$ were the upper limit ages for the spectral base of ID 120 and ID 146, respectively. The synthetic base consists of a set of 12 spectra with ages of 0.1 , 1.0, 5.0, 9.0 and 10.0 Gyr and metallicities of $Z=0.05,0.02$ (solar) and 0.004 . Note that we have chosen this range of metallicities because it was only possible to measure a limited number of Lick indices in our spectra. The synthesis results for ID 120 and ID 146 are presented in Table 3 as the percentual contribution of each template to the normalized spectrum, and the $E(B-V)$ values obtained from the synthesis. Figs 5 and 6 show the spectra corrected for reddening and the synthesized population spectra constructed by the sum of the base spectra according to the proportions given by the synthesis. According to the stellar population synthesis, the galaxy ID 120 has an important contribution of young stellar populations (0.1 Gyr), about 47 per cent. Despite this contribution of a younger population, it is still made up of about 32 per cent of an old stellar population (10 Gyr) and the remaining is of intermediate-age stellar population. A very large reddening of $E(B-V)=0.82$ was obtained from the synthesis. A solar metallicity is a better fit for this galaxy. In a general way, the estimates of old, intermediate and young stellar populations for ID 120 are in agreement with those derived by Serote Roos et al. (2005) for field galaxies at intermediate redshifts.

For the galaxy ID 146 its spectrum is best fitted by the template set of subsolar metallicity $(Z=0.004)$ with a dominant contribution of almost 60 per cent of an old stellar population $(10 \mathrm{Gyr})$ and

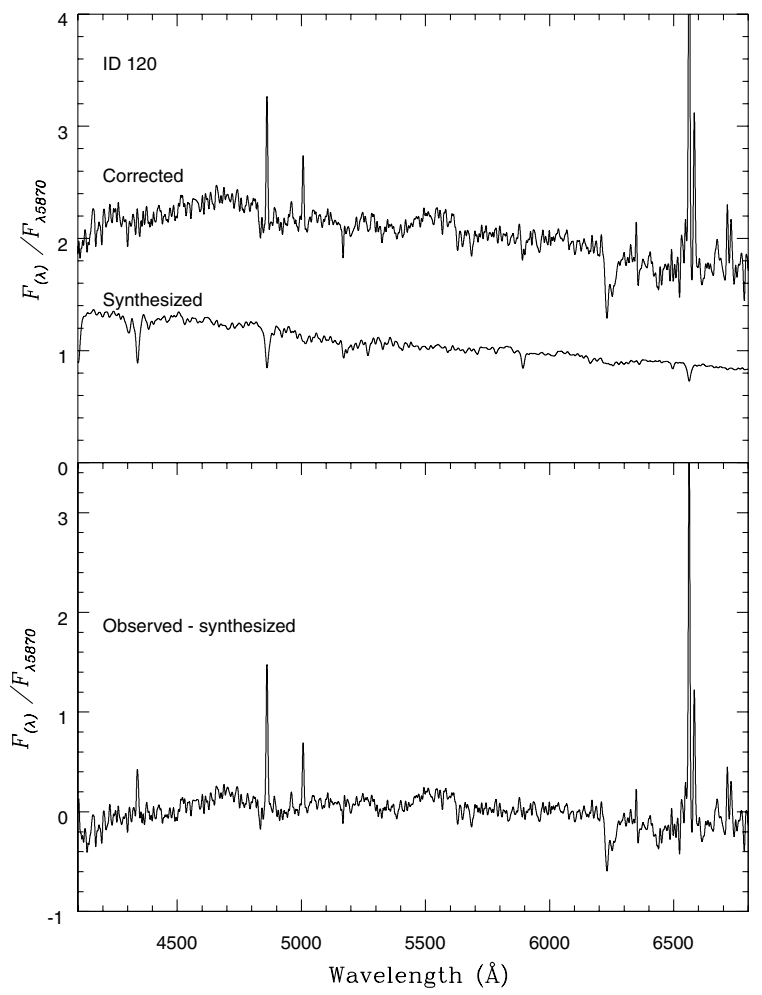

Figure 5. Stellar population synthesis for ID 120. Top panel: spectrum corrected for reddening and the synthesized spectrum. Bottom panel: pure emission spectrum. The corrected spectrum has been shifted by a constant.

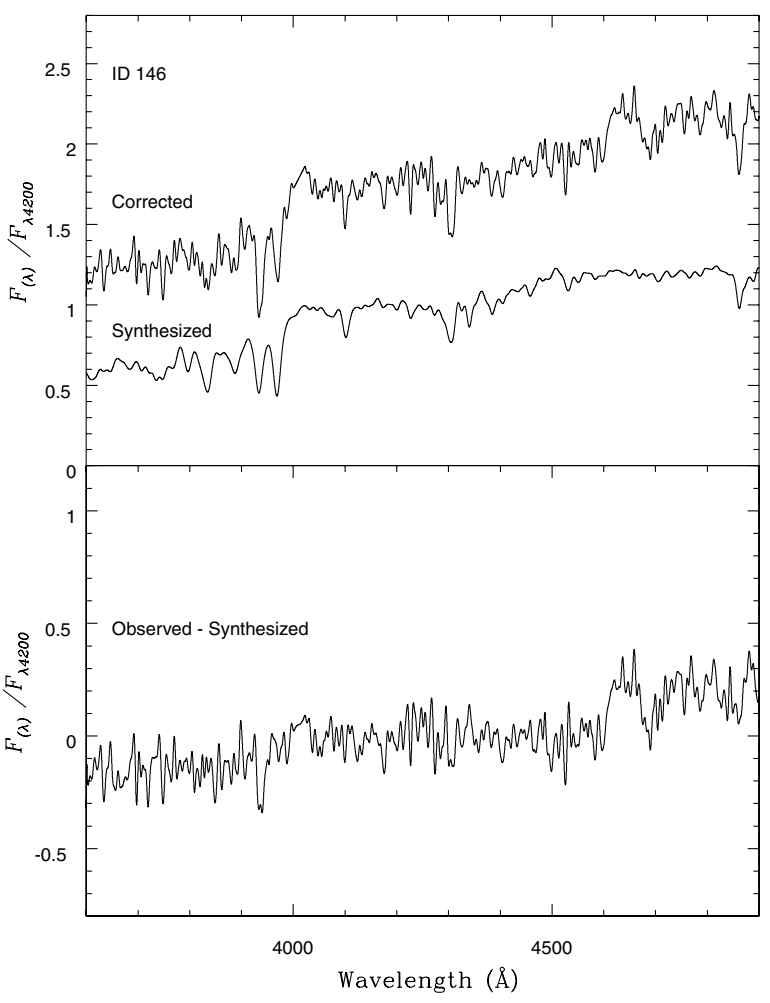

Figure 6. Stellar population synthesis for ID 146. Top panel: spectrum corrected for reddening and synthesized spectrum. Bottom panel: spectrum without the stellar component The corrected spectrum has been shifted by a constant. 
a significant contribution of about 19 and 17 per cent from intermediate components of 1 and $5 \mathrm{Gyr}$, respectively. Also, a young $(0.1 \mathrm{Gyr})$ stellar population was marginally detected $(\approx 5$ per cent $)$. The synthesis indicates a notable reddening in the spectrum, $E(B-$ $V)=0.90$.

Differences between the stellar population of field galaxies and cluster members have been reported by Serote Roos et al. (2005). These authors have performed stellar population synthesis in the cluster members of CL $0048-2942(z \sim 0.64)$ as well as in the field galaxies $(0.2229<z<0.6287$ and $0.6502<z<0.8215)$ and have found in a general way that field galaxies seem to host less-evolved stellar populations than cluster members. Unfortunately, since we can only derive the stellar population for two galaxies, ID 120 and ID 146, we cannot compare the stellar populations of field galaxies and cluster LCDCS-S001 members.

\section{IONIZED GAS}

As discussed earlier, the spectrum of the galaxy ID 120 presents conspicuous emission lines. In this section, we investigate the physical properties and the ionization source of the gas in this galaxy.

First, we subtracted the contribution of the stellar population from the spectrum using the results of the stellar population synthesis. We then estimated the line intensities using Gaussian line profile fitting procedures, which are listed in Table 4.

In order to identify the main excitation source of the gas in ID 120 we used the standard diagnostic diagrams with emission-line ratios of easily observed lines, such as the classical diagrams of $\log ([\mathrm{O}$ III] $\lambda 5007 / \mathrm{H} \beta)$ versus $\log ([\mathrm{N}$ II $] \lambda 6584 / \mathrm{H} \alpha)$ and $\log ([\mathrm{O}$ III $] \lambda 5007 / \mathrm{H} \beta)$ versus $\log ([\mathrm{S} \mathrm{II}] \lambda \lambda 6717,6731 / \mathrm{H} \alpha)$ (Veilleux \& Osterbrock 1987). They are applied either for nearby galaxies (Pastoriza, Donzelli \& Bonatto 1999) or for galaxies at higher redshifts (Maier et al. 2005; Lamareille et al. 2006a). In these diagnostic diagrams, the emissionline ratios of ID 120 are located in an $\mathrm{H}$ II region zone.

We derived the electron density $N_{\mathrm{e}}$ from the [S II] $\lambda 6716 / \lambda 6731$ intensity ratio by solving numerically the equilibrium equations for an $n$-level atom using the TEMDEN routine of the NEBULAR package of STSDAS/IRAF assuming an electron temperature of $10000 \mathrm{~K}$. The energy levels, transition probabilities and collisional strength values for [S II] were taken, respectively, from Bowen (1960), Keenan et al. (1993) and Ramsbottom, Bell \& Stafford (1996). A value of $N_{\mathrm{e}}=$ $377 \pm 184 \mathrm{~cm}^{-3}$ was estimated, which is compatible with the ones derived in the range of $N_{\mathrm{e}} \approx 30-400 \mathrm{~cm}^{-3}$ by Puech et al. (2006) in six galaxies at $z=0.55$ and are typical values of classical galactic $\mathrm{H}$ II regions (Copetti et al. 2000) as well as of giant extragalactic H II regions (Castaneda, Vilchez \& Copetti 1992).

Considering that the emission lines of ID 120 are produced in an $\mathrm{H}$ II region we have employed the photoionization code CLOUDY/96.03 (Ferland 2002) to produce a model of H II region that reproduces the observed emission-line intensities. Basically, the input nebular parameters in the models are the metallicity $Z$, ionization parameter $U$, upper stellar mass limit $M_{\text {up }}$, age $A$ and electron density $N_{\mathrm{e}}$. The $M_{\text {up }}, A$ and $\log (U)$ were chosen to be initially $40 \mathrm{M}_{\odot}$,

Table 4. Predicted and observed relative line fluxes $(\mathrm{H} \beta=100)$.

\begin{tabular}{lcc}
\hline Line & Observed & Model \\
\hline$[\mathrm{O}$ III] $\lambda 5007$ & $41.95 \pm 1.97$ & 48.00 \\
{$\left[\mathrm{~N}_{\text {II }}\right] \lambda 6584$} & $98.50 \pm 4.24$ & 98.00 \\
{$\left[\mathrm{~S}_{\mathrm{II}}\right] \lambda 6717$} & $36.18 \pm 2.02$ & 36.00 \\
{$\left[\mathrm{~S}_{\mathrm{II}}\right] \lambda 6731$} & $32.61 \pm 3.61$ & 33.00 \\
\hline
\end{tabular}

$2 \mathrm{Myr}$ and -2.5 , respectively, and were changed interactively until a suitable solution was found, following the same fitting procedures as adopted by Dors \& Copetti (2006) (see description details in this paper). Besides, we have assumed in our models an electron density of $N_{\mathrm{e}}=377 \mathrm{~cm}^{-3}$ and solar metallicity - since the synthesis results indicate that the spectrum of ID 120 is better fitted with this metallicity.

We found an excellent agreement between the observational data and the model of $12+\log (\mathrm{O} / \mathrm{H})=8.65, M_{\text {up }}=100 \mathrm{M}_{\odot}$, and age of $5.5 \mathrm{Myr}$ (see Table 4). The oxygen abundance derived is compatible with the solar composition of $12+\log (\mathrm{O} / \mathrm{H})=8.69$ estimated by Allende Prieto, Lambert \& Asplund (2001) and with measurements of abundance derived for other star-forming galaxies at intermediate redshift (Barrientos, Schade \& Lopez-Cruz 1996; Contini et al. 2002; Lilly, Carollo \& Stockton 2003; Lamareille et al. 2006b; Mouhcine et al. 2006). For example, Mouhcine et al. (2006) derived oxygen abundances for a sample of 40 luminous field galaxies with redshifts in the range of $0.22<z<0.8$ and found that their $12+\log (\mathrm{O} / \mathrm{H})$ values are ranging from 8.4 to 9.0 , with a median of 8.7 . Besides, these studies about metallicity in galaxies at intermediate redshift have confirmed that the relation between luminosity and metallicity found in these galaxies is very similar to the relation obtained for galaxies in the local Universe. The values of $12+\log (\mathrm{O} / \mathrm{H})=8.65$ and $M_{B}=-18.75$ derived for ID 120 are compatible with the values of luminosity and metallicity for galaxies at intermediate redshifts found by these authors.

\section{CONCLUSIONS AND FINAL REMARKS}

We present spectroscopic and photometric analysis for eight field galaxies in the direction of the galaxy cluster LCDCS-S001. The photometric and spectroscopic data were obtained with the GMOS instrument in the Gemini South Observatory and consists of an $i^{\prime}$ band image for the cluster field and spectra centred at $7500 \AA$. The main findings are the following.

(i) Redshifts of $z=0.7464$ and 0.7465 were determined for the field galaxies ID 440 and ID 461, respectively. For the other six galaxies we have confirmed the redshift calculated in Paper I. The redshifts of the field galaxies are in the range of $0.2201<z<0.7784$.

(ii) Stellar velocity dispersions were measured for five field galaxies and were found to be in the range of $200<\sigma<346 \mathrm{~km} \mathrm{~s}^{-1}$.

(iii) Blue luminosities were calculated for six galaxies which are brighter than $M_{B}=-18.64$.

(iv) The galaxies ID 180, ID 266, ID 428 and ID 461 follow the Faber-Jackson relation and therefore are early type. In these galaxies we did not detect a brightening of the $B$ luminosity with respect to the local galaxies.

(v) Lick indices were measured for ID 120 and ID 146 and used to determine their stellar population properties, by means of spectral synthesis. In a general way, we found that ID 146 has a dominant contribution of an old stellar population $(\approx 10 \mathrm{Gyr})$ of subsolar metallicity. ID 120 presents a spectrum with both emission and absorption lines, having an important contribution, of about 47 per cent, of young stellar population and a significant contribution of about 32 per cent of older population $(\approx 10 \mathrm{Gyr})$. The spectrum of ID 120 is better fitted with solar metallicity. Large reddening values of $E(B-V)=0.90$ and 0.82 were derived for ID 120 and ID 146, respectively.

(vi) According to the classical diagnostic diagrams, the emission lines present in the ID 120 spectrum are produced in an $\mathrm{H}$ II region. 
An electron density of $N_{\mathrm{e}}=377 \pm 184 \mathrm{~cm}^{-3}$ was derived from the [S II] $\lambda 6716 / \lambda 6731$ ratio.

(vii) The values of $12+\log (\mathrm{O} / \mathrm{H})=8.65$ and $M_{B}=-18.75$ derived for ID 120 are compatible with the values of luminosity and metallicity found by other authors for galaxies at intermediate redshifts.

\section{ACKNOWLEDGMENTS}

We thank the referee, Dr Florence Durret, for helpful comments and suggestions. This work has been partially supported by the Brazilian institution CNPQ.

\section{REFERENCES}

Allende Prieto C., Lambert D. L., Asplund M., 2001, ApJ, 556, L63

Barrientos L. F., Schade D., Lopez-Cruz O., 1996, ApJ, 460, L89

Bica E., 1988, A\&A, 195, 9

Bowen I. S., 1960, ApJ, 132, 1

Bruzual G., Charlot S., 2003, MNRAS, 344, 1000

Butcher H., Oemler A. Jr., 1984, ApJ, 285, 426

Castaneda H. O., Vilchez J. M., Copetti M. V. F., 1992, A\&A, 260, 370

Contini T., Treyer M. A., Sullivan M., Ellis R. S., 2002, MNRAS, 330, 75

Copetti M. V. F., Mallmann J. A. H., Schmidt A. A., Castañeda H. O., 2000, A\&A, 357, 621

Dors O. L. Jr., Copetti M. V. F., 2006, A\&A, 452, 473

Dressler A., Lynden-Bell D., Burstein D., Davies R. L., Faber S. M., Terlevich R. J., Wegner G., 1987, ApJ, 313, 42

Faber S. M., Jackson R. E., 1976, ApJ, 204, 668

Ferland G. J., 2002, Hazy, a Brief Introduction to Cloudy 96.03, Univ. Kentucky Dept. Phys., Astron. Internal Report

Fritz A., Ziegler B. L., Bower R. G., Smail I., Davies R. L., 2005, MNRAS, 358,233
Keenan F. P., Hibbert A., Ojha P. C., Conlon E. S., 1993, Phys. Scr. A, 48, 129

Kormendy J., 1977, ApJ, 217, 406

Lamareille F., Contini T., Le Borgne J.-F., Brinchmann J., Charlot S., Richard J., 2006a, A\&A, 448, 893

Lamareille F., Contini T., Brinchmann J., Le Borgne J.-F., Charlot S., Richard J., 2006b, A\&A, 448, 907

Lilly S. J., Carollo C. M., Stockton A. N., 2003, ApJ, 597, 730

Maier C., Lilly S. J., Carollo C. M., Stockton A., Brodwin M., 2005, ApJ, 634,849

Mouhcine M., Bamford S. P., Aragón-Salamanca A., Nakamura O., MilvangJensen B., 2006, MNRAS, 369, 891

Nakamura O., Aragón-Salamanca A., Milvang-Jensen B., Arimoto N., Ikuta C., Bamford S. P., 2006, MNRAS, 366, 144

Pastoriza M. G., Donzelli C. J., Bonatto C., 1999, A\&A, 347, 55

Poggianti B. M., Smail I., Dressler A., Couch W. J., Barger A. J., Butcher H., Ellis R. S., Oemler A. J., 1999, ApJ, 518, 576

Puech M., Flores H., Hammer F., Lehnert M. D., 2006, A\&A, 455, 131

Ramsbottom C. A., Bell K. L., Stafford R. P., 1996, Atom. Data Nucl. Data Tables, 63, 57

Rembold S. B., Pastoriza M. G., 2007, MNRAS, 374, 1056 (Paper I)

Schmitt H. R., Bica E., Pastoriza M. G., 1996, MNRAS, 278, 965

Serote Roos M., Lobo C., Durret F., Iovino A., Márquez I., 2005, A\&A, 429, 101

Trager S. C., Worthey G., Faber S. M., Burstein D., Gonzalez J. J., 1998, ApJS, 116, 1

Treu T., Stiavelli M., Casertano S., Møller P., Bertin G., 2002, ApJ, 564, L13 van Dokkum P. G., Franx M., Kelson D. D., Illingworth G. D., 2001, ApJ, 553, L39

Veilleux S., Osterbrock D. E., 1987, ApJS, 63, 295

Worthey G., Ottaviani D. L., 1997, ApJS, 111, 377

Ziegler B. L., Bender R., 1997, MNRAS, 291, 527

This paper has been typeset from a $\mathrm{TE}_{\mathrm{E}} \mathrm{L} / \mathrm{ET} \mathrm{X}$ file prepared by the author. 